\title{
Seroprevalencia de Leptospirosis en Trabajadores de Limpieza Pública del Distrito de San Juan Bautista, Ayacucho
}

\author{
Seroprevalence of Leptospirosis in Public Cleaning Workers of the \\ District of San Juan Bautista, Ayacucho \\ Claudia Quispe-Girón ${ }^{1,3}$, Eddy Cabrera-Bellido², Felipe Achallma-Vilca ${ }^{2}$, \\ Magaly Rodríguez Monje ${ }^{1}$, Gloria Betty Adrianzen Facundo ${ }^{1}$
}

\section{Resumen}

El objetivo del presente trabajo fue determinar la seroprevalencia y factores asociados de la leptospirosis humana en trabajadores de limpieza pública del distrito de San Juan Bautista, Ayacucho, Perú. Se realizó una encuesta epidemiológica y las muestras de sangre fueron analizadas por ELISA IgM y microaglutinación (MAT) para la determinación de serovares de Leptospira. De los 41 trabajadores examinados, 25 (62.5\%) fueron varones y $16(37.5 \%)$ fueron mujeres. Tres trabajadores resultaron positivos $(7.32 \%)$, dos varones y una mujer. Ninguno de los posibles factores de riesgo fue estadísticamente significativo. Los tres casos positivos presentaron títulos de 1/3200, 1/1600 y 1/3200 contra el serovar Varillal.

Palabras clave: leptospirosis, trabajadores de limpieza pública, Perú

\section{ABstract}

The aim of this study was to determine the prevalence and associated factors of human leptospirosis in public cleaning workers of the district of San Juan Bautista, Ayacucho, Peru. An epidemiological survey was conducted and blood samples were analyzed by ELISA IgM and agglutination (MAT) for the determination of Leptospira serovars. Of the 41 workers tested, $25(62.5 \%)$ were male and $16(37.5 \%)$ were women.

\footnotetext{
${ }^{1}$ Escuela de Medicina Veterinaria, Facultad de Ciencias Agrarias, Universidad Nacional San Cristóbal de Huamanga, Ayacucho, Perú

${ }^{2}$ Centro de Salud San Juan Bautista Ayacucho, Ayacucho, Perú

${ }^{3}$ E-mail: claudia.quispe.g@upch.pe
} 
Three workers resulted positive (7.32\%), two men and one woman. None of the possible risk factors was statistically significant. The titres of the three positive cases were 1/3200, $1 / 1600$ and 1/3200 against serovar Varillal.

Key words: leptospirosis, public cleaning workers, Peru

\section{INTRODUCCIÓN}

La leptospirosis es una enfermedad infecciosa de distribución mundial y de comportamiento endémico en muchos países, que puede alcanzar niveles de epidemia (Pappas et al., 2008). La infección es causada por bacterias del género Leptospira y se presenta, mayormente, en zonas urbanas y rurales que presentan deficiencias en salubridad (Johnson, 2004).

En el Perú, el primer caso de leptospirosis fue diagnosticado en la ciudad de Lima en un ciudadano japonés (Arce y Ribeyro, 1917). El contagio se produce con mayor frecuencia por el contacto directo con la orina de un animal infectado, o con agua o ambientes contaminados con orina infectada, especialmente en lugares húmedos y protegidos de la luz que favorece la supervivencia de la bacteria (MINSA, 2011). Reportes en la ciudad de Ayacucho entre 2009 y 2011 indican que hay un incremento de 4 a 29 casos de personas con leptospirosis; no obstante, en dicha ciudad, al igual que en otras de la región, llegan a los hospitales muchas personas con enfermedades e infecciones sin que se llegue de determinar de manera concluyente los agentes etiológicos que las causen (ASIS-DIRESA, 2011).

El riesgo de adquirir infecciones en los trabajadores de limpieza pública y en aquellos que manejan residuos es mayor debido a su exposición a desechos que pueden contener excretas y secreciones de animales (López et al., 2015). Las enfermedades laborales u ocupacionales son el resultado de infecciones latentes en la comunidad que predisponen a trabajadores frente a enfermeda- des (Palomino, 2011). Por ello, el objetivo del presente estudio fue determinar la seroprevalencia y factores asociados de la leptospirosis humana en trabajadores de limpieza pública del distrito de San Juan Bautista, Ayacucho.

\section{Materiales y Métodos}

\section{Características del Estudio}

El estudio fue observacional de tipo descriptivo y de corte transversal. Se realizó en el distrito de San Juan Bautista, localizado en la provincia de Huamanga, departamento de Ayacucho, Perú, a una altitud promedio de $2800 \mathrm{msnm}$.

La población evaluada fueron los trabajadores de limpieza pública de la municipalidad distrital $(n=41)$. En este grupo había trabajadores de áreas verdes $(n=3)$, dedicados a la limpieza y mantenimiento de parques y plazas; trabajadores de limpieza de urbana $(n=32)$, encargados de la recolección de residuos sólidos de calles y veredas, y los choferes del vehículo recolector $(n=6)$. La toma, remisión y entrega de resultados fue con total confidencialidad y reserva de la identidad de los involucrados.

Se coordinó con el Centro de Salud San Juan Bautista, con el Área de Salud Ambiental y Zoonosis y con la Subgerencia de Servicios de la Municipalidad de San Juan Bautista para la ejecución del estudio. Se reunió a los trabajadores de limpieza pública y se les explicó las características de la leptospirosis y el propósito del estudio. Una vez lograda la aprobación del personal de limpieza, se reali- 
zó una encuesta estructurada por el Instituto Nacional de Salud (INS), la cual contiene datos generales y epidemiológicos.

Se tomaron muestras de sangre siguiendo la técnica convencional y las normas de bioseguridad (INS, 2002). Primeramente, se trabajó con un grupo de 21 personas y en la semana siguiente se obtuvieron las 23 muestras restantes, con el fin de ajustarse a la disponibilidad de tiempo de los trabajadores. Se recolectó $7 \mathrm{ml}$ de sangre venosa de cada participante, utilizando tubos al vacío sin anticoagulante. Se dejaron las muestras en reposo por cerca de $30 \mathrm{~min}$, y se centrifugaron a $349 \mathrm{~g}$ durante $20 \mathrm{~min}$. Los sueros fueron transferidos a crioviales estériles de $2 \mathrm{ml}$ y conservados a $4-8^{\circ} \mathrm{C}$ hasta su procesamiento. Cada vial fue rotulado con un código correspondiente a cada persona (INS, 2002).

Las muestras fueron procesadas en el Laboratorio Regional de Salud Pública de la Dirección Regional de Salud, Ayacucho, determinando la presencia de anticuerpos antiLeptospira, mediante la prueba de ELISA indirecta IgM. Se empleó el kit de ELISA IgM elaborado por el Instituto Nacional de Salud (INS) del Perú. Las muestras y fichas epidemiológicas de cada paciente con los resultados de la prueba de ELISA fueron enviadas al Laboratorio Nacional de Referencia de Metaxénicas Bacterianas del Instituto Nacional de Salud, Lima, para su serotipificación y confirmación de resultados mediante la técnica de microaglutinación (MAT).

La encuesta permitió estratificar la información proporcionada para las siguientes variables: edad (18-30, 31-43, 44-46, 5770 ), sexo, grado de instrucción (primaria, secundaria, superior), ocupación (las tres actividades indicadas anteriormente), zona de vivienda (urbana, peri-urbana), recojo de residuos sólidos (adecuado, deficiente), destino de residuos sólidos (adecuado, deficiente), abastecimiento de agua (adecuado, deficiente), destino de excretas (adecuado, defi- ciente). El resultado fue analizado mediante la prueba del Chi cuadrado.

Se determinó la seroprevalencia de leptospirosis humana, mediante el programa SPSS 23 para Windows para crear una base de datos con la información epidemiológica.

\section{Resultados}

En el grupo de 41 trabajadores evaluados, $25(62.5 \%)$ eran varones y $16(37.5 \%)$ eran mujeres. Los resultados indicaron la ocurrencia de tres personas positivas a leptospirosis, dos varones y una mujer, resultando en una seroprevalencia de $7.32 \%$. En los casos positivos, se encontraron títulos de MAT de 1/3200, 1/1600 y 1/3200 contra el serovar Varillal.

El promedio de edad de los trabajadores fue de 43.2 años, con una desviación estándar de 11.4 años. El análisis estadístico con los tres casos positivos en la población de 41 trabajadores no logró identificar factores de riesgo.

\section{Discusión}

E1 $7.38 \%$ de seroprevalencia para leptospiras en trabajadores de limpieza pública en el distrito de San Juan Bautista, Ayacucho, fueron similares al 8.6\% (29/339) de seroprevalencia reportado en trabajadores de servicio de aseo urbano de la municipalidad de Asunción, Paraguay (López et al., 2015), así como al 8.9\% (27/302) encontrado por Palomino (2011) en los manipuladores de alimentos de los mercados de abastos del distrito de Ayacucho, Perú.

Otras investigaciones realizadas en condiciones y ambientes diferentes presentan resultados diversos. Así, Platts et al. (2006) reportó 1.2\% (3/250) en habitantes del distrito de Puente Piedra, Lima, zona que presenta deficiencias de salubridad que favorecen 
la transmisión de leptospirosis; en tanto que Vargas et al. (2005) encontró el 30.6\% de seroprevalencia de anticuerpos antiLeptospira en pacientes con síndrome febril en el Hospital de Apoyo San Francisco, en Ayna, Ayacucho.

No se pudo determinar posibles factores de riesgo, posiblemente debido a la limitada población en estudio y baja prevalencia. No obstante, en el estudio de López et al. (2015), la única variable que se relacionó significativamente con la seropositividad de los trabajadores fue la de trabajar en el área de recolección de residuos domiciliarios (16.3\%). Por otro lado, Palomino (2011), evaluando manipuladores de alimentos en los mercados de abastos de Ayacucho, determinó que los factores de riesgo para el contagio fueron el contacto con aguas servidas y desagües $(\mathrm{OR}=1.42)$, contacto con orina de roedores $(\mathrm{OR}=1.2)$, contacto con carnes de porcinos y vacunos contaminados $(\mathrm{OR}=1.15)$ y si sus alimentos son consumidos por las ratas $(\mathrm{OR}=3.8)$.

Los títulos de MAT variaron entre 1/1600 y 1/3200 contra el serovar Varillal. Vargas et al. (2005), en pacientes febriles que acuden al Hospital de Apoyo San Francisco en Ayna, Ayacucho, encontraron 15 serovares de Leptospira, siendo los más frecuentes icterohaemorrhagiae (título de 1/800), djasiman (título de 1/800), varillal (título de $1 / 800$ ) y panama (título de $1 / 1600$ ).

La norma técnica para la atención integral de la leptospirosis humana del Instituto Nacional de Salud (INS, 2002) indica que para la prueba de Microaglutinación (MAT) se requiere de 2 a 3 muestras, con intervalos de 15 días. Sin embargo, cuando solo se dispone de una muestra, un título igual o superior a 1:800 en la región de la selva del Perú, podría estar confirmando el diagnóstico. Asimismo, títulos menores (1:100 a 1:400) confirman el diagnóstico en zonas costeras y en la sierra del Perú. La región de Ayacucho se encuentra en la zona de la sierra centro sur, de allí que los títulos obtenidos corresponden a casos positivos.
El estudio permitió establecer la seroprevalencia de anticuerpos antiLeptospira para la población de trabajadores de limpieza pública de Ayacucho, la cual puede considerarse como de relativa mediana prevalencia. Es importante promover el uso de equipos de protección personal, mascarilla, guantes y botas, así como mejorar las medidas preventivas y de promoción de la salud.

\section{Conclusiones}

- La seroprevalencia de anticuerpos antileptospirosis en los trabajadores de limpieza pública del distrito de San Juan Bautista, Ayacucho, fue de $7.32 \%$.

- No se encontraron factores asociados a la positividad a anticuerpos antiLeptospira en estos trabajadores.

\section{Agradecimientos}

Los autores expresan su agradecimiento al Fondo Nacional de Desarrollo Científico, Tecnológico y de Innovación Tecnológica (FONDECYT) por el financiamiento del proyecto: Maestría en Sanidad Acuícola en la Universidad Peruana Cayetano Heredia Convenio N. ${ }^{\circ}$ 230-2015-FONDECYT- Esquema Financiero EF 023 «Programa de Maestría en Universidades Peruanas».

\section{Literatura Citada}

1. Arce JY, Ribeyro RE. 1917. Sobre un caso de espiroquetosis icterohemorrágica. Cron Med (Lima) 34: 355-360.

2. [ASIS-DIRESA] Dirección Regional de Salud Ayacucho, Dirección de Epidemiología, Emergencias y Desastres. Ministerio de Salud. 2001. Análisis de la situación de salud de Ayacucho2011. [Internet]. Disponible en: http:// www.bvsde.paho.org/documentosdigitales/bvsde/texcom/ASIS-regiones/Ayacucho/Ayacucho2011.pdf 
3. Céspedes M, Ormaeche M, Condori P, Balda L, Glenny M. 2003. Prevalencia de leptospirosis y factores de riesgo en personas con antecedentes de fiebre en la provincia de Manu, Madre de Dios, Perú. Rev Peru Med Exp Salud Pública 20: 80-185.

4. [INS] Instituto Nacional de Salud. 2002. Norma técnica para la atención integral de la leptospirosis humana. [Internet]. Disponible en: http:// www.ins.gob.pe/repositorioaps/0/4/jer/$1 / 1$ eptopirosis/normat $\%$ c $3 \%$ 89 cnicaparala\%20atenci\%c-3\%93nintegral de la leptospirosis humana.pdf

5. Johnson MA, Smith H, Joseph P, Gilman RH, Bautista CT, Campos KJ, et al. 2004. Environmental exposure and leptospirosis, Peru. Emerg Infect Dis 10: 1016-1022. doi: 10.3201/eid1006.030660

6. López F, Samudio M, de Assis MD, Cabello A. 2015. Seroprevalencia de leptospirosis y factores asociados en trabajadores del servicio de aseo urbano de la Municipalidad de Asunción, Paraguay. Rev Chil Infectol 32: 628-633. doi: 10.4067/S0716-10182015000700003

7. [MINSA] Ministerio de Salud. 2011. Prevención para la salud. Lima. [Inter- net]. Disponible en: http://www. minsa.gob.pe/index.asp?op=2

8. Platts-Mills J, LaRochelle P, Campos K, Vinetz J, Gotuzzo E, Ricaldi J. 2006. Seroprevalencia de leptospirosis en Puente Piedra, Lima en el año 2006. Rev Peru Med Exp Salud Publica 28: 273-276. doi: 10.1590/S1726-46342011000200014

9. Palomino S. 2011. Seroprevalencia de leptospirosis en manipuladores de alimentos de los mercados de abastos del distrito de Ayacucho, 2010. Tesis de Biólogo. Ayacucho, Perú: Univ Nacional San Cristóbal de Huamanga. 60 p.

10. Pappas G, Papadimitriou P, Siozopoulou V, Christou L, Akritidis N. 2008. The globalization of leptospirosis: worldwide incidence trends. Int J Infect Dis 12: 351-735. doi: 10.1016/ j.ijid.2007.09.011

11. Vargas F, García V, Céspedes M, Palomino M, Ayala T. 2008. Seroprevalencia y factores asociados con leptospirosis en pacientes con síndrome febril en Ayacucho, Perú, 2005. Rev Perú Med Exp Salud Pública 25: 190-194. doi: 10.17843/rpmesp.2008.252.1257 